\title{
Prevalence of Hepatitis in Paediatric Oncologic Patients: A Single Centre Study
}

\author{
Yusra Alam ${ }^{*}$ Zille Huma and Nizam-ul-Hassan \\ Child Aid Association, National Institute of Child Health, Karachi, Pakistan.
}

\begin{abstract}
Background: Oncologic problems are increasing day by day very rapidly. It includes leukaemia, solid tumors, germ cell tumors and many others. Leukaemia is a growing reason of paediatric mortality. Children with leukaemia, usually have low blood count which requires blood transfusions. These transfusions can lead to transfusion associated viral infections particularly Hepatitis B and Hepatitis C.
\end{abstract}

Objective: To identify the prevalence rate of $\mathrm{HBV}$ and $\mathrm{HCV}$ infections in paediatric oncology patients and to identify the probable relation of transfusion with that of the spread of the infection and its detection method.

Methods: This cross-sectional study was carried out at molecular department of Child Aid Association Karachi from January 2013 to December 2015. A sample size of 200 paediatric oncology patients was taken. The samples were processed by Real Time Polymerase Chain Reaction (PCR) technique.

Result: The results showed that out of 200 patients, 17 were HBV positive having a prevalence rate of $8.5 \%$ and 69 were HCV positive with prevalence rate of $34.5 \%$. Among $17 \mathrm{HBV}$ positive patients, no patient was infected before the start of treatment while 03 out of $69 \mathrm{HCV}$ positive patients were previously infected.

Conclusion: The incidence of HCV and HBV is raising in the paediatric oncology patients on treatment. This demonstrates that the management may be responsible for this predicament. These patients are exposed to cytotoxic and chemotherapeutic drug infusions and multiple blood transfusions. Therefore blood transfusion practices should meet the standard operating procedures which ensure the use of sterilized equipments and the donors should be screened for viral hepatitis through PCR.

Keywords: Hepatitis B, Hepatitis C, Paediatric Oncologic, PCR.

doi.org/10.21089/njhs.21.0008

\section{INTRODUCTION}

Oncological problems are becoming a nightmare now days. These include leukaemia, solid tumors, germ cell tumors and many others. Leukaemia is found to be one of the leading causes of paediatric mortalities. Children with leukaemia usually have low blood count hence requiring frequent blood transfusions. These multiple transfusions can lead to transfusion associated viral infections particularly Hepatitis B (HBV) and Hepatitis C (HCV) infections [1]. Also stated that children with haematological disorders have high risks of infection with both hepatitis B (HBV) and C (HCV) viruses and prevalence rates are as high as $50 \%$ in leukaemia and lymphoma patients.

\section{Hepatitis B Infection}

Hepatitis B virus (HBV) is a blood-borne and sexually transmitted virus. Each year, 600,000 HBV-related deaths

*Address correspondence to this author at the Child Aid Association NICH, Karachi, Pakistan;

E-mail: yusra_alam88@yahoo.com occur worldwide, most of which result from the chronic sequelae of HBV infection. Approximately $25 \%$ of persons who become chronically infected during childhood and $\sim 15 \%$ of those who become chronically infected after childhood die from cirrhosis or liver cancer [2].

\section{Hepatitis C Infection}

Hepatitis C virus (HCV) is the most common cause of hepatitis after blood transfusion, which may result in severe liver damage leading to liver transplantation. Children who have had multiple transfusions as a result of chronic anaemia, cancer, haemophilia, received extracorporeal membrane oxygenation or long-term parenteral nutrition, are at high risk for $\mathrm{HCV}$ infection. The reported prevalence ranges from $10 \%-85 \%$ percent $[3,4]$ also found $\mathrm{HCV}$ as the most common aetiology of chronic hepatitis, cirrhosis, and hepatocellular carcinoma.

\section{Epidemiology}

The World Health Organization (WHO) estimated that 2 billion people worldwide, have a serologic evidence of past 
or present HBV infections and 360 million are chronically infected and at risk for HBV-related liver disease. Approximately, one third of all cases of cirrhosis and half of all cases of hepatocellular carcinoma can be attributed to chronic HBV infection. HBV is estimated to be responsible for $500,000-700,000$ deaths each year [4]. WHO estimated that approximately $3 \%$ of the world population has been infected with HCV. There are around 170 million patients with HCV in the world, and 3 - 4 million individuals are diagnosed as new cases every year. Approximately 2 million cases are in Japan, 2.7 million are in the United States, 5 million are in Europe and approximately 10 million are in Pakistan. Approximately, a quarter of a million deaths per annum occur due to chronic liver disease associated with HCV. [5] Lavanchy [6] also found that, more than 500,000 new cases of liver cancer occur each year, among which 22\% (>100 000) are attributable to HCV infection. Prospective studies have shown that $80 \%$ of cases of acute hepatitis $C$ progress to chronic infection; $10-20 \%$ of these will develop complications of chronic liver disease, such as liver cirrhosis, within two to three decades of onset, and 1-5\% will develop liver cancer, making $\mathrm{HCV}$ a health problem of global importance.

\section{Transmission}

Over the last decade in the developed world, all donated blood products have been screened for both HBV and HCV, which has led to a major reduction in post transfusion viral hepatitis. However, in developing countries, these screening assays were introduced at a later stage and were partially implemented, which has led to an increased risk of acquiring both HBV and HCV infections in such countries [7].

HBV infections are mostly acquired perinatally or during early childhood in approximately $45 \%$ of the world's population who live in regions that are highly endemic for HBV infection. Another $43 \%$ live in regions of intermediate endemicity, where multiple modes of transmission (i.e., perinatal, household, sexual, injection drug use associated, and health care associated) are important. In countries of low endemicity, most infections occur among adolescents and adults and are attributable to sexual and injection drug use exposures [2]. Furthermore, it has also been stated by Raja that, blood transfusion remains the main mode of transmission of $\mathrm{HCV}$ infection, as unscreened blood and its products are still in use in many developing countries instead of the utilisation of modern screening techniques [3].

Initially, it was believed that blood transfusion and intravenous drug abuse were the most common routes of transmission of HCV. Further epidemiological studies revealed the existence of other possible modes of transmission of the disease were present which included unsafe injections (reuse of glass syringes or needles), vertical transmission, non-sexual contact in households, face or armpit shaving at community barber shops, ear piercing, tattooing and inadequately sterilized surgical or dental instruments. [1,3] also identified that both in the developed and under developed countries, there have been nosocomial outbreaks in the paediatric populations due to improper implementation of universal precautions such as reuse of disposable materials, incorrect handling of sterile materials, through person to person contact and through invasive procedures.

\section{Prevention}

Early attempts to screen the blood for hepatitis viruses have markedly reduced the transfusion-related infection rate [5] [1] also favored Raja and said that the development of blood donor screening tests for the virus has remarkably declined the transmission of HCV but there are many patients, who are surviving with chronic transfusion-acquired infection. However, some patients resolve the infection spontaneously, while most remain chronically infected, which progresses to liver cirrhosis or hepatocellular carcinoma.

The most common methods used for the detection of the viruses are serological, involving indirect detection of antibodies against the viruses using enzyme immunoassay (EIA) systems for initial screening, followed by a confirmation test with a recombinant immune-blot assay or similar second- or third generation assay. In some immune-suppressed children, the anti-HCV assay appears negative due to their disease pattern and/or due to treatment but the child is actually infected with HCV. In these cases, it is necessary to retest the children by using a different diagnostic approach, such as a molecular assay which can determine the presence of $\mathrm{HCV}$ RNA [1].

To prevent Hepatitis, a great deal of credit for achievements to date, stems from the introduction of hepatitis B vaccines. First licensed in the United States in 1981, hepatitis B vaccine is now one of the most widely used vaccines in the world and is part of the routine vaccination schedule for many of the world's infants and children. It is the world's first cancer and sexually transmitted disease preventive vaccine. In countries where large-scale vaccination efforts were made in the first decade after introduction of the vaccine, the epidemiology of hepatitis B and HBV infection has been transformed, and there are early signs that the burden of HBV-related sequelae will be significantly reduced as vaccinated populations age [7]. Also supported Hepatitis B vaccination as the most effective measure to prevent HBV infection and its consequences. However, for persons already infected with HBV, antiviral agents are available that may prevent the serious sequelae of chronic liver disease, which highlighted the importance of identifying infected individuals [2].

By reviewing all of the above facts, the objective of the study is to identify the prevalence rate of $\mathrm{HBV}$ and $\mathrm{HCV}$ 
infections in paediatric oncology patients and to identify the probable relation of transfusion with that of the spread of the infection and its detection method.

\section{MATERIALS AND METHODS}

\section{Study Subject and Design}

This cross-sectional study was carried out at Molecular department of Child Aid Association, NICH, Karachi, from January 2013 to December 2015. A sample size of 200 oncology patients was taken. Broadly, the samples were divided into two groups after detection of the viruses, while the positive samples were further divided into three groups; transfusion/reuse needles/tattoo history of patients, baseline hepatitis infections and diagnostic methods for analysis.

\section{Inclusion Criteria}

All children under 17 yrs of age, diagnosed with any type of malignancy were included in the study. Some already had positive reactions in serology while the rest had baseline investigation before the start of the treatment.

\section{Detection Technique}

The samples were processed by real-time polymerase chain reaction (RT-PCR) technique. In this technique, first viral RNA is extracted from the blood sample then it gets amplified in the PCR machine. After amplification, the machine gives the replicative count of the virus present in the sample in numeric form which is considered as the quantitative detection of the infection.

\section{RESULT}

\section{Prevalence and Characteristics of Patients}

The results showed that, out of 200 patients, 86 had viral hepatitis. Among 86 patients, 17 were found with HBV hav- ing a prevalence rate of $8.5 \%$ and 69 were $\mathrm{HCV}$ positive with a prevalence rate of $34.5 \%$ (Fig. 1).

\section{Transfusion/Reuse Needles History and Infection}

Out of $17 \mathrm{HBV}$ positive patients, 47\% (08) had history of transfusion / reuse needles / tattoos, while 32\% (22) had a similar history among $69 \mathrm{HCV}$ positive patients (Fig. 2).

\section{Baseline Infection among Patients}

Before admission, out of 17 , none of the patient was previously HBV infected, while 03 out of 69 patients with $\mathrm{HCV}$ infection were previously reactive (Fig. 3).

\section{Importance of Diagnostic Method}

Out of $17 \mathrm{HBV}$ patients, 02 were detected on ICT and the rest were positive on PCR. However, 11 patients were detected on ICT, 01 on ELISA and the rest 57 were diagnosed with HCV reactivity on PCR (Fig. 4).

\section{DISCUSSION}

The results showed that the prevalence rate of $\mathrm{HCV}$ is much higher than HBV as Lavanchy [6] also found that, among 500,000 new cases of liver cancer each year, about $22 \%$ $(>100$ 000) are attributable to HCV infection only. This prevalence of $\mathrm{HCV}$ can be dangerous as it could worsen the outcome of successfully treated paediatric oncology patients because of a fact that about $80 \%$ of cases of acute hepatitis $\mathrm{C}$ progress to chronic infection; $10-20 \%$ of these will develop complications of chronic liver disease, such as liver cirrhosis, within two to three decades of onset, and $1-5 \%$ will sis, within two to three decades of onset, and 1-5\% will develop liver cancer [6]. While discussing the infection pattern, $47 \%$ patients of $\mathrm{HBV}$ and $32 \%$ of $\mathrm{HCV}$ patients have history of transfusions before the start of oncological treatment.

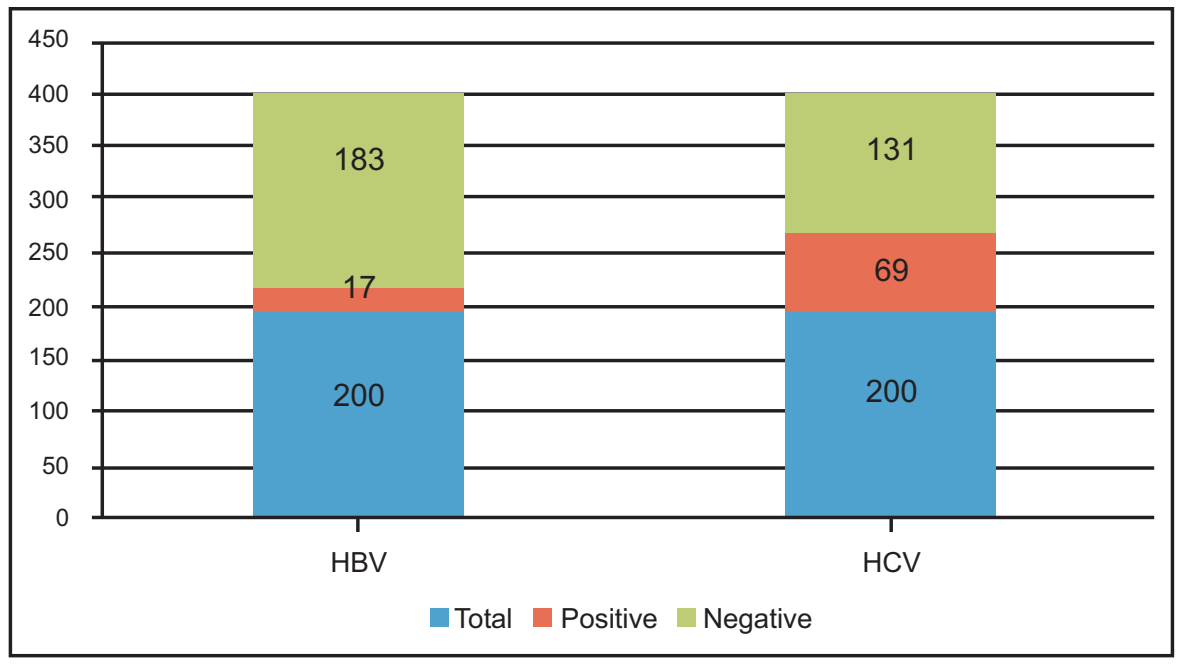

Fig. (1). Patients Characteristics. 


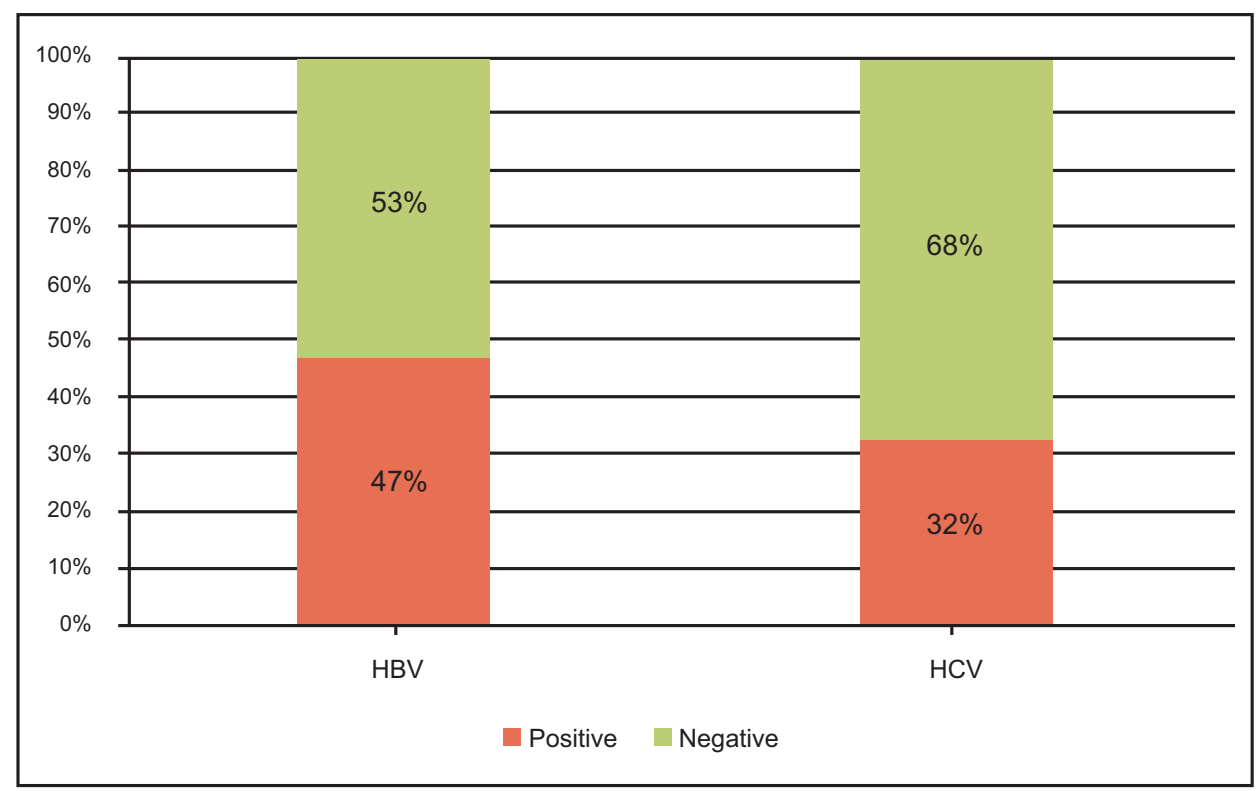

Fig. (2). Transfusion/reuse needles history.

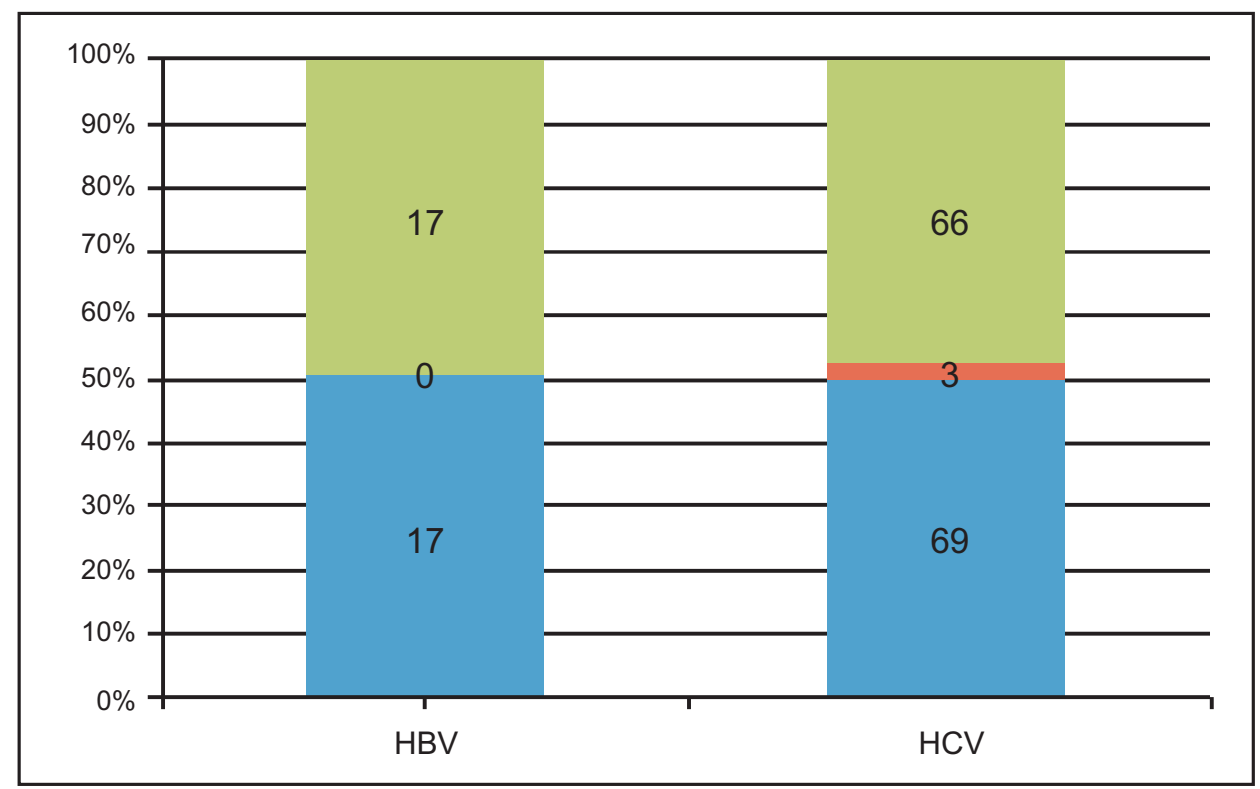

Fig. (3). Baseline Infection.

As patients have undergone multiple transfusions before and during the treatment, therefore, it can be a cause of spreading infection. Furthermore, no patient of HBV was affected before the start of treatment while only 03 patients out of 69 were previously affected which shows that a significant number of patients were getting infection during the oncologic treatment cycle, in which they receive multiple transfusions. It has also been stated by [5] as well that, blood transfusion remains the main mode of transmission of $\mathrm{HCV}$ infec- tion, as unscreened blood and its products are still in use in many developing countries instead of the utilisation of modern screening techniques. While discussing the diagnostic method of hepatitis, majority of the patients were negative on ICT and ELISA but they all were positive on PCR which shows the sensitivity of the method as [1] also stated that although the most common methods used for the detection of the viruses are serological, involving indirect detection of antibodies against the viruses using enzyme immunoassay 
(EIA) systems for initial screening, followed by a confirmation test with a recombinant immune-blot assay but in some immune-suppressed children, the anti-HCV assay appears negative due to their disease pattern and/or due to treatment but the child is actually infected with HCV. In these cases, it is necessary to retest the children by using a different diagnostic approach, such as a molecular assay which can determine the presence of HCV RNA.

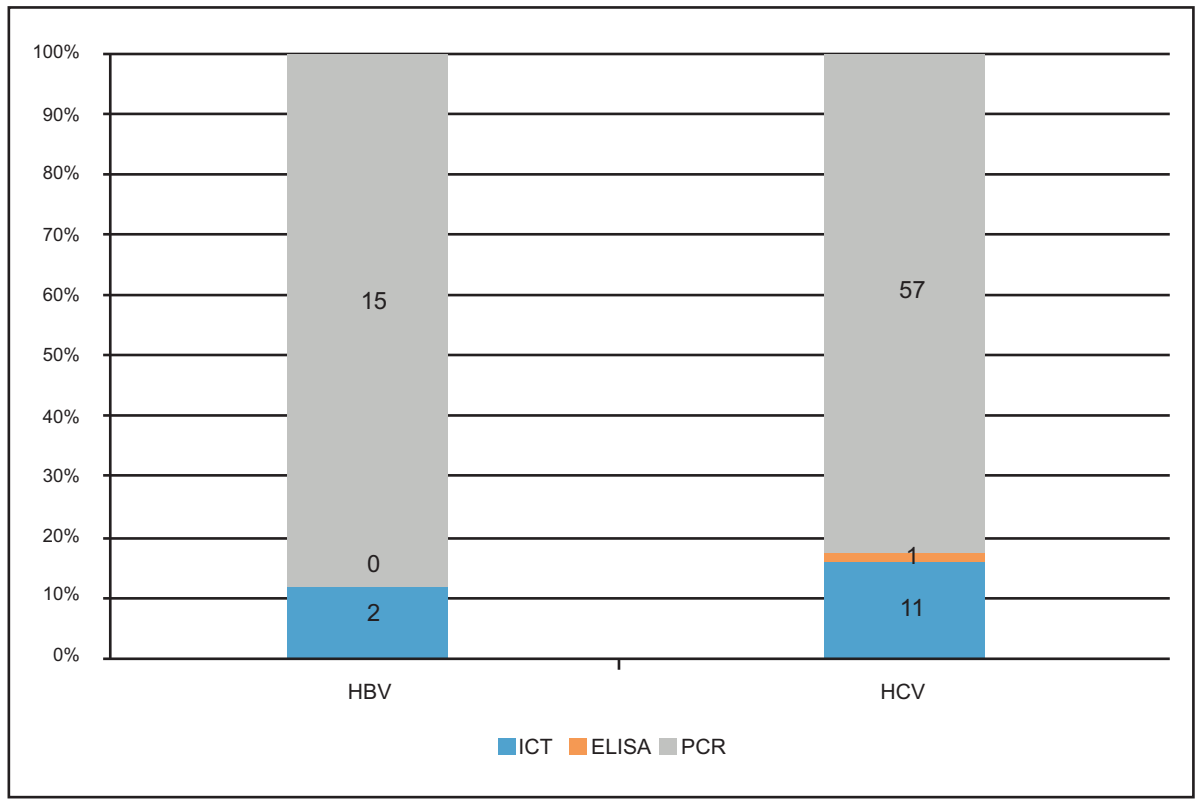

Fig. (4). Diagnostic method.

\section{CONCLUSION}

The study concludes that the incidence of HCV and HBV is increasing in the paediatric oncology patients on treatment. This demonstrates that their management may be responsible for this predicament. These patients are exposed to cytotoxic and chemotherapeutic drug infusions and multiple blood transfusions. Therefore blood transfusion practices should meet the standard operating procedures which ensure the use of sterilized equipments and the donors should be screened for viral hepatitis through PCR.

\section{LIMITATIONS}

Following are the main limitations of the study:

- All data is totally based on the information written on the request form given to the molecular department and in patient file.

- Incomplete information is due to improper documentations.

- Results might vary from organization to organization.

- $\quad$ Reliability and validity might be compromised due to small sample size.
- Study was carried out based mainly on the transfusion history, while there are other aetiologies as well for the occurrence of the infection.

\section{ACKNOWLEDGEMENT}

Declared none.

\section{CONFLICT OF INTEREST}

Declared none.

\section{REFERENCES}

[1] Visoná K, Baez F, Taylor L, Berr'ios R, León B, Pacheco C, et al. Impact of Hepatitis B and Hepatitis C Virus Infections in a Hematology-Oncology Unit at a Children's Hospital in Nicaragua, 1997 to 1999. Clin. Diag. Lab. Immunol., 2002; (9)3: 622-626, 1071-412X/02/\$04.000 DOI: 10.1128/CDLI.9.3.622-626.2002.

[2] Wasley A, Kruszon-Moran D, Kuhnert W, Simard EP, Finelli L, McQuillan G, et al. The prevalence of hepatitis B virus infection in the united states in the era of vaccination. J. Infect. Dis., 2010; 202(2): 192-201.

[3] Vogt M, Lang T, Frösner G, Klingler C, Endl AFS, Zeller A, et al. Prevalence and clinical outcome of hepatitis $\mathrm{C}$ infection in children who underwent cardiac surgery before the implementation of blood-donor screening. New Engl. J. Med., 1999; 341: 866-870.

[4] Castellino S, Lensing S, Riely C, Rai SN, Davila R, Hayden RT, et al. The epidemiology of chronic hepatitis $\mathrm{C}$ infection in survivors 
of childhood cancer: an update of the St Jude Children's Research hospital hepatitis C seropositive cohort. Blood. 2004; 103: 7.

[5] Raja NS and Janjua KA. Epidemiology of hepatitis C virus infection in Pakistan. J. Microbiol. Immunol. Infect., 2008; 41: 4-8.

[6] Lavanchy D. Evolving epidemiology of hepatitis C virus. Clin. Microbiol. Infect., 2010 - 2011; 17: 107-115.
[7] Shepard CW, Simard EP, Finelli L, Fiore AE, and Bell BP. Hepatitis B virus infection: epidemiology and vaccination. Epidemiol. Rev., 2006; 28:112-125.

Received: May 11, 2016

Revised: October 25, 2016

Accepted: December 29, 2016

(C) 2017 National journal of health sciences.

This is an open-access article. 